\title{
Editorial
}

\section{Normal and abnormal function of the diaphragm}

The diaphragm and the other inspiratory muscles form a vital pump. The ancient Greeks recognised this and thought that the diaphragm was the seat of the soul. Indeed the Greek word for soul is phrenes and this is how the phrenic nerves got their name. The action of the diaphragm has been a source of controversy for centuries, and the twentieth is no exception. It has long been recognised that when the diaphragm contracts it not only pushes downward on the abdominal contents but also exerts an upward force on the rib cage into which its fibres are inserted. Quantification of its action on the rib cage is the source of the controversy.

Goldman and Mead proposed that the pressure which displaced the rib cage during diaphragmatic contraction in upright man was abdominal pressure. ${ }^{1}$ How this could be the case is explained in the figure which is a simple model of the respiratory system. The muscular part of the diaphragm is represented by the muscle fibres extending from the rib cage to the bar which represents the central tendon of the diaphragm. Contraction of the diaphragm exerts a downward force on the central tendon and thus the abdomen. It also exerts an equal and opposite upward force on the rib cage, so that the force displacing the abdomen and rib cage are identical. Because it is abdominal pressure which displaces the abdomen, abdominal pressure can also be thought of as displacing the rib cage. To the extent that this is a realistic model of the respiratory system, it gives insight into how abdominal pressure could be equivalent to the pressure displacing the rib cage. However, Goldman and Mead's suggestion remains an unproven hypothesis which only applies to man in the upright posture. In the supine posture, diaphragmatic contraction has little action on the rib cage. For this reason, in the upright posture both rib cage and abdomen are displaced during quiet breathing, whereas in the supine posture most of the motion is abdominal. Breathing movements are not different between men and women. ${ }^{2}$

Address fcr reprint requests: Dr PT Macklem, Meakins-Christie Laboratories, McGill University Clinic, Department of Medicine, Royal Victoria Hospital, Montreal, Canada.
If one wishes to determine the contribution of the diaphragm to breathing relative to the other inspiratory muscles, the key is abdominal pressure. Contraction of the diaphragm increases abdominal pressure and displaces the abdomen outward. Contraction of the other inspiratory muscles alone produces a decrease in abdominal pressure and displaces the abdomen inward. The fall in pleural pressure produced by intercostal and accessory muscle contraction which inflates the lung, is transmitted across the flaccid diaphragm to the

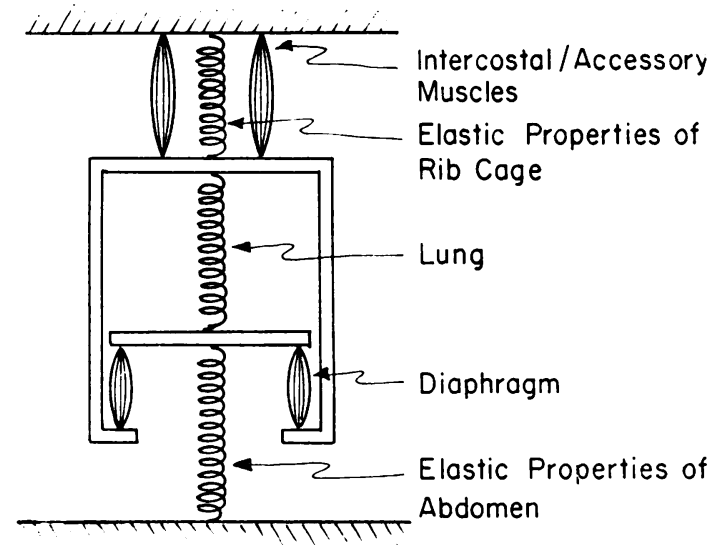

Figure Simplified model of the respiratory system. The rib cage is represented as a bar in the shape of an inverted $U$. Its elastic properties are represented by the upper spring. The diaphragmatic fibres are inserted into the lower margin of the rib cage and into the central tendon of the diaphragm represented by a horizontal bar. The middle spring represents the lung and the lower spring, the elastic properties of the abdomen. Diaphragmatic contraction exerts a force upward on the rib cage which is equal and opposite to the force it exerts downward on the abdomen. Intercostal/assessory muscle contraction inflates the rib cage, shortening the upper spring, and inflates the lung. However it displaces the abdomen in the expiratory direction by lengthening the lower spring. Combined diaphragmatic and intercostal/accessory muscle contraction may lengthen, shorten, or produce no displacement of the abdomen depending upon the relative strength of contraction of the two muscle groups. 
abdomen. The resulting fall in abdominal pressure and inward abdominal displacement is expiratory, and is a most inefficient way to breathe. Simultaneous contraction of the diaphragm and other inspiratory muscles results in a change in abdominal pressure which depends upon the relative strength of contraction of the two muscle groups. Thus, provided the abdominal muscles remain relaxed during inspiration, the change in abdominal pressure and the resulting displacement of the abdomen gives valuable information on the relative contributions of the diaphragm and intercostal/accessory muscles to inspiration.

The normal range of breathing movements at rest and during exercise is not precisely known but probably ranges from predominantly abdominal, as in normal supine man at rest, to predominantly rib cage in conditions in which the intercostal and accessory inspiratory muscles are recruited to the extent that there is little abdominal motion.

Although the limits of normal motion are not well described, inward inspiratory displacements of the abdomen are definitely abnormal and represent a displacement every bit as paradoxical as inward inspiratory displacements of the rib cage. Abdominal paradoxical motion is observed in bilateral diaphragmatic paralysis and diaphragmatic fatigue, and is a valuable clinical sign indicating serious diaphragmatic malfunction. Of course inward abdominal displacements can result from abdominal muscle contraction, and it is important clinically to distinguish whether the abdomen is sucked in because of weak diaphragmatic contraction or pushed in as a result of contraction of the abdominal muscles. Palpation of the abdomen readily distinguishes between the two.

Prominent rib cage motion in the supine position is also an abnormal breathing pattern and signifies recruitment of other inspiratory muscles. This probably occurs in any condition which leads to a sufficient increase in the work of breathing.

These physical signs have only recently been described. ${ }^{3-7}$ It is surprising, to say the least, that a system as vital as the inspiratory muscles has received so little attention from chest physicians. We know much about the lung, but little about the muscles which pump it. We know much about the physiological properties of skeletal muscles in general, but little about the diaphragm and other inspiratory muscles in particular. When other skeletal muscles perform work it is usually against inertial loads. However when the inspiratory muscles work they usually have to cope with elastic and resistive loads. In diseases characterised by decreased compliance or increased airways resistance these loads can become excessive and may lead to fatigue. Yet we know little about how elastic and resistive loads influence muscle function. We have only the vaguest idea of the energy requirements of the respiratory muscles in diseases which increase the work of breathing and no clear idea of how these requirements are met or even if they are met. For instance, is it possible when there is a failure of the oxygen transport system, as $\overrightarrow{\vec{x}}$ in cardiogenic shock, that the oxygen demands of the inspiratory muscles deprive the rest of the $\omega$ body of much needed oxygen-or does the rest of $\vec{\sigma}$ the body deprive the inspiratory muscles of the energy they need? Some of these questions are beginning to be answered. Aubier and colleagues $\vec{T}$ recently studied the action of the diaphragm in 3 cardiogenic shock caused by cardiac tamponade ${ }^{8}$ 号 (and personal communication). By injecting saline into the pericardium they were able to reduce $\infty$ cardiac output in dogs in a highly reproducible. fashion. In dogs receiving artificial ventilation, there was no mortality, whereas in dogs breathing spontaneously mortality was high. Death invariably occurred from failure of inspiratory muscle contraction while the heart continued to beat. In $\triangle$ spite of an increase in the amplitude of the phrenic $\overrightarrow{\overrightarrow{0}}$ neurogram (an index of the central drive to 3 breathe) and a similar increase in the amplitude of the diaphragmatic EMG (an index of diaphragmatic excitation), the transdiaphragmatic pressure (an index of the force of contraction) diminished. Clearly these results indicate that there was failure ? of excitation/contraction coupling or of the contractile machinery, and this was the immediate cause of death.

These important findings raise many questions. How commonly does this occur clinically, if ever? Under what circumstances can we predict failure $\frac{7}{2}$ of the inspiratory muscles? What is the exact mechanism that leads to failure or fatigue? What o pharmacological agents can influence the con- $N$ tractile properties of the respiratory muscles and $\mathrm{N}$ protect against fatigue? Should the indications for artificial ventilation be expanded to treat or protect against fatigue?

But perhaps the most important question is: where have we respirologists been all these years? In 1919, Davies, Haldane and Priestley produced inspiratory muscle fatigue in normal subjects by $\overrightarrow{\mathbb{D}}$ adding external resistances to the airway. Although $\frac{\mathbb{D}}{\mathbb{Q}}$ they wrongly attributed their results to fatigue of the respiratory centres they pointed out that "the phenomena in question are of great impor- 
tance in practical medicine. They throw a new light on failure of the respiratory centre as a common - perhaps the most common-immediate cause of death, and on directions towards which therapeutic measures can be directed." Davies, Haldane and Priestley realised that failure of the vital inspiratory pump was lethal, common, and important. We have had for many years the technology to study it. Why didn't we? How could we have so successfully forgotten Davies', Haldane's and Priestley's message? This is a real puzzler. Even today how many respirologists have a working knowledge of the mechanisms of excitationcontraction coupling? How many respirologists make any attempt to assess inspiratory muscle function? Or treat it?

The normal and abnormal function of the inspiratory muscles is belatedly becoming a major area of clinical research. This is a welcome and long overdue development. By correlating simple clinical observations such as the inspiratory movements of rib cage and abdomen with more basic experiments which give greater understanding of impaired or improved contractility of the diaphragm and other inspiratory muscles, much valuable information will be gained. For this reason the paper by De Troyer and Estenne on Limitations of measurement of transdiaphragmatic pressure in detecting diaphragmatic weakness in this issue of Thorax is important. Such contributions may alter radically the diagnosis and management of cardiorespiratory disorders.

PT MACKLEM
Meakins-Christie Laboratories
McGill University
Montreal
Canada

\section{References}

1 Goldman M, Mead J. Mechanical interaction between the diaphragm and rib cage. J Appl Physiol 1973; 35:197-204.

2 Sharp JT, Goldberg NB, Druz WS, Danon J. Relative contributions of rib cage and abdomen to breathing in normal subjects. J Appl Physiol 1975; 39:608-18.

3 Ashutosh K, Gilbert R, Auchincloss JH, Peppi D. Asynchronous breathing movements in patients with obstructive pulmonary disease. Chest 1975; 67:553-7.

4 Pontoppidan H, Geffin B, Lowenstein E. Acute respiratory failure in the adult. $N$ Engl $J$ Med 1972; 287:743-52.

5 Sharp JT, Goldberg NB, Druz WS, Fishman HC, Danon J. Thoracoabdominal motion in chronic obstructive pulmonary disease. Am Rev Respir Dis 1977; 115:47-56.

6 Derenne J-Ph, Macklem PT, Roussos Ch. State of the Art. The respiratory muscles: mechanics, control and pathophysiology. Part III. Am Rev Respir Dis 1978; 118:581-601.

7 Newsom-Davis J, Goldman M, Gasson M. Diaphragm function and alveolar hypoventilation. Q J Med New Series 1976; XLV: 87-100.

8 Aubier M, Viires N, Sillye G, Roussos Ch. Effect of mechanical ventilation on lactic acidosis in shock. Am Rev Respir Dis 1980; 121:311.

9 Davies HW, Haldane JS, Priestley JG. The response to respiratory resistance. J Physiol (Lond) 1919; 53: 60-9. 